\title{
Properties of SH-SAWs in Layered Piezomagnetic/Piezoelectric Structures covered in a microbeam array
}

\author{
Ruixin $\mathrm{Jin}^{1}$, Benjie Ding ${ }^{2}$, Xian Fu² and Lili Yuan ${ }^{1,2^{*}}$ \\ ${ }^{1}$ School of Architectural, Civil Engineering and Environment, Ningbo University, 818 Fenghua Road, \\ Ningbo, Zhejiang 315211, China \\ ${ }^{2}$ School of Mechanical Engineering and Mechanics, Ningbo University, 818 Fenghua Road, Ningbo, \\ Zhejiang 315211, China \\ yuanlili@nbu.edu.cn
}

Keywords: SH-SAWs, piezomagnetic, piezoelectric, microbeam; dispersion

Abstract. This paper investigates the shear horizontal surface acoustic wave (SH-SAWs) propagating in layered piezomagnetic/piezoelectric structures covered a microbeam array, which involves a thin piezomagnetic layer bonded perfectly to an unbounded piezoelectric substrate. The beams are modeled by the Euler-Bernoulli theories when they are bending during the piezomagnetic/piezoelectric structures in shear motion. The explicit formulations of SH-SAWs dispersion relations in such layered structure with consideration of magnetoelectrically open and shorted cases are derived. The effects of the microbeam array on the phase velocity are analyzed. The numerical results are presented and discussed. From the results, we can find that the effects of microbeams on the properties of the SH-SAWs are remarkable. The phase velocity decrease with the non-dimensional wave number and increase with the number of microbeams and the thickness of piezomagnetic layer. The analytical method and the results in the paper could be useful to the design of the chemical sensors of magnetoelectric materials.

\section{Introduction}

It is well known that the composite materials consisting of piezoelectric (PE) and piezomagnetic (PM) have the ability to convert energy between magnetic and electric fields which is not present in single-phase piezoelectric or piezomagnetic materials [1-2]. For this reason, PM-PE composites are potential candidates for magnetic sensors, transducers and microwave devices, e.g. delay lines, magnetic field probes, damage detectors, medical ultrasonic imaging, and resonators, etc. These applications are done with wave propagations and vibrations in PM-PE composites, and so their dynamic behavior is the first concern in design as well as in performance. A number of investigations have been reported about the wave propagation problems in such composites [3-4].

We all known a large number micro- or nano-scale beam arrays have been made by using different techniques due to the extensive effort on micro- and nano-technologies [5-6]. These new structures have great potentials for new devices such as efficient microneedles, dynamic tuning of surface wetting, microelectromechanical systems (MEMS) actuators and resonators, etc. There are strong needs to study the effects of microbeams on vibrations of structures or wave propagation in structures due to the design of resonators, sensors and actuators. Li et al discussed the collective buckling of nonuniform nanobeams interacting through an elastic substrate [7]. Lin et al considered the effects of surface deformation on the collective buckling of an array of rigid beams on an elastic substrate [8]. Liu et al investigated shear vibration of a rotated Y-cut quartz crystal plate carrying an array of microbeams with their bottoms fixed to the top surface of the plate [9]. From the paper, we can find that the plate was modeled by the theory of anisotropic elasticity and the beams were modeled by the Euler-Bernoulli theory for beam bending, and a frequency equation that determines the resonant frequencies of the structure was derived. However, there is no report about the shear horizontal surface acoustic waves (SH-SAWs) propagating in layered piezomagnetic/piezoelectric structures covered a microbeam array.

In this paper, the propagation of SH-SAWs in layered piezomagnetic/piezoelectric structures 
carrying an array of microbeams is investigated. The microbeams are modeled by the Euler-Bernoulli theories when they are bending during the piezoelectric structures in shear motion. The dispersion relations are calculated and discussed in detail.

\section{Formulation of the problem}

The layered magnetoelectric structure carrying an array of microbeams illustrated as Figure.1, where a half-space piezoelectric substrate is covered by a piezomagnetic thin layer.

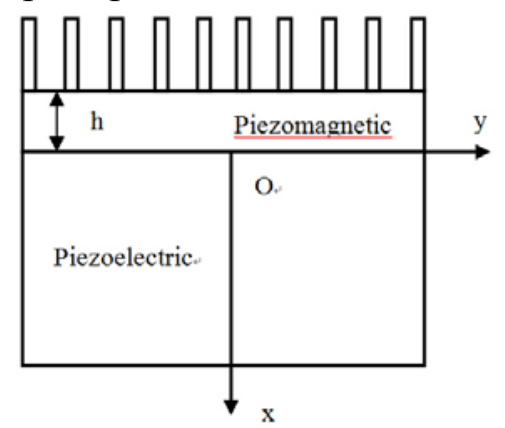

Figure 1. A piezoelectric half space with a piezomagnetic layer covered a microbeam array

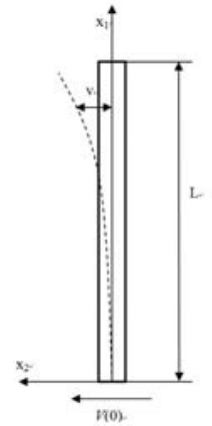

Figure 2. Notation and coordinate system for beam bending

The piezomagnetic and piezoelectric materials are polarized along the $z$-direction. We here only consider the so-called anti-plane wave propagation problem. The displacement components, the electric potential and the magnetic potential are assumed as

$$
u(x, y, t)=0, v(x, y, t)=0, w=w(x, y, t), \phi=\phi(x, y, t), \psi=\psi(x, y, t),
$$

where $u, v, w$ are the displacement components in $x, y$ and $z$ direction, respectively; $\phi$ is electric potential, and $\psi$ is magnetic potential.

For linearly magnetoelectric materials, the equilibrium equations of elasticity without body forces and the Gauss's law of magneto-electrostatics without free charge are given as follows $\sigma_{j i, j}=\rho \ddot{u}_{i}, D_{i, i}=0, B_{i, i}=0, i, j=1,2,3$,

where $\sigma_{i j}, D_{i}$ and $B_{i}$ are the stress, electric displacement and magnetic induction (i.e., magnetic flux) components, respectively, $\rho$ is the mass density. The subscript comma denotes a partial derivative with respect to the coordinates, and a superimposed dot represents the derivative with respect to the time.

For an anisotropic and linearly magneto-electro-elastic solid, the coupled constitutive relation can be written as

$$
\begin{aligned}
& \sigma_{i}=c_{i k} \gamma_{k}-e_{k i} E_{k}-f_{k i} H_{k}, \\
& D_{i}=e_{i k} \gamma_{k}+\varepsilon_{i k} E_{k}+g_{i k} H_{k}, \\
& B_{i}=f_{i k} \gamma_{k}+g_{i k} E_{k}+\mu_{i k} H_{k},
\end{aligned}
$$

where $\gamma_{i}, E_{i}$, and $H_{i}$ are the strain, electric field, and magnetic field components, respectively; $c_{i j}$, $\varepsilon_{i j}$ and $\mu_{i j}$ are the elastic, dielectric, and magnetic permeability coefficients, respectively; $e_{i j}, f_{i j}$ and $g_{i j}$ are the piezoelectric, piezomagnetic, and magneto-electric coefficients, respectively. Substituting the equations (1), (3) into equations (2), the coupling wave equations and the constitutive equations can be obtained as

$$
\begin{aligned}
& c_{44} \nabla^{2} w+e_{15} \nabla^{2} \phi+f_{15} \nabla^{2} \psi=\rho \frac{\partial^{2} w}{\partial t^{2}}, \\
& e_{15} \nabla^{2} w-\varepsilon_{11} \nabla^{2} \phi-g_{11} \nabla^{2} \psi=0, \\
& f_{15} \nabla^{2} w-g_{11} \nabla^{2} \phi-\mu_{11} \nabla^{2} \psi=0,
\end{aligned}
$$


where $\nabla^{2}=\frac{\partial^{2}}{\partial x^{2}}+\frac{\partial^{2}}{\partial y^{2}}$ is the two-dimensional Laplacian operator in the Cartesian coordinates.

\section{Equations and fields of the beam}

For the flexural motion of the beams, we use the Euler-Bernoulli theory of bending $[10,11]$. By calculating, we can get the shear force at the bottom as

$V(0)=C E I f^{\prime \prime \prime}(0, E I, \hat{\rho} A, L, \omega) \exp [i k(y-c t)]$,

\section{Continuity conditions and boundary conditions}

The continuity conditions at the interface between the thin piezomagnetic layer and the piezoelectric substrate are written as

$$
\begin{array}{ll}
w^{e}(0, y, t)=w(0, y, t), & \sigma_{x z}^{e}(0, y, t)=\sigma(0, y, t), \\
\phi^{e}(0, y, t)=\phi(0, y, t), & D_{x}^{e}(0, y, t)=D(0, y, t), \\
\psi^{e}(0, y, t)=\psi(0, y, t), & B_{x}^{e}(0, y, t)=B(0, y, t) .
\end{array}
$$

Assume the number density of the beams per unit area of the elastic surface is N. There are two typical cases being considered in this study, namely, the magneto-electrically open and the sh orted surface conditions. The magneto-electrically open conditions are written as

$$
\begin{aligned}
& \sigma_{x z}(-h, y, t)=N V(0), \phi(-h, y, t)=\phi^{0}(-h, y, t), w(-h)=-v(0), \\
& \psi(-h, y, t)=\psi_{0}(-h, y, t), D_{x}(-h, y, t)=D_{0}(-h, y, t), B_{x}(-h, y, t)=B_{0}(-h, y, t) .
\end{aligned}
$$

The magneto-electrically shorted conditions are given as

$$
\begin{aligned}
& \sigma_{x z}(-h, y, t)=N V(0), w(-h)=-v(0), \\
& \phi(-h, y, t)=0, \psi(-h, y, t)=0 .
\end{aligned}
$$

\section{Solution of the problem}

We consider the displacement, electric potential and magnetic potential of Eqs. (4) of piezomagnetic layer as

$$
\begin{aligned}
& w(x)=\left[A_{1} \cos (k b x)+A_{2} \sin (k b x)\right] e^{i k(y-c t)}, \\
& \phi(x)=\left[A_{3} \mathrm{e}^{k x}+A_{4} \mathrm{e}^{-k x}\right] e^{i k(y-c t)}, \\
& \psi(x)=\left[\frac{f_{15}}{\mu_{11}} A_{1} \cos (k b x)+\frac{f_{15}}{\mu_{11}} A_{2} \sin (k b x)+A_{5} \mathrm{e}^{k x}+A_{6} \mathrm{e}^{-k x}\right] e^{i k(y-c t),}
\end{aligned}
$$

where $b=\sqrt{\rho c^{2} / \bar{c}_{44}-1}$ and $\bar{c}_{44}=c_{44}+\frac{f_{15}^{2}}{\mu_{11}^{m}} . A_{1}, A_{2}, A_{3}, A_{4}, A_{5}$ and $A_{6}$ are unknowns to be determined.

The displacement, electric potential and magnetic potential of piezoelectric substrate are given as

$$
\begin{aligned}
& w^{e}(x)=A_{1}^{e} e^{-k b^{e} x} e^{i k(y-c t)}, \\
& \psi^{e}(x)=A_{2}^{e} e^{-k x} e^{i k(y-c t)}, \\
& \phi^{e}(x)=\left[\frac{e_{15}}{\varepsilon_{11}^{e}} A_{1}^{e} e^{-k b^{e} x}+A_{3}^{e} \mathrm{e}^{-k x}\right] e^{i k(y-c t),}
\end{aligned}
$$


where $b^{e}=\sqrt{1-\rho^{e} c^{2} / \bar{C}_{44}^{e}}$ and $\bar{c}_{44}^{e}=c_{44}^{e}+\frac{e_{15}^{2}}{\varepsilon_{11}^{e}} . A_{1}^{e}, A_{2}^{e}$ and $A_{3}^{e}$ are unknown constants.

\section{Solutions of equations}

Inserting Eqs. (12), (13) and their corresponding components of stress and displacement into boundary and continuity conditions, we can obtain the algebraic equations of the unknowns

$$
[\mathbf{A}][C]=0 \text {. }
$$

In order to obtain the nontrivial solutions of the above-mentioned unknown constants, the determinant of the coefficient matrix of these linear algebraic equations must equal zero, i.e. $|A|=0$. So the dispersive relations of the magneto-electrically open and shorted case can be obtained.

\section{Numerical results}

For the numerical calculations, the piezomagnetic material is assumed to be $\mathrm{CoFe}_{2} \mathrm{O}_{4}$, and the piezoelectric substrate material is $\mathrm{BaTiO}_{3}$. Unless otherwise specified, the thickness of piezomagnetic layer is assumed to be $h=0.1 \mathrm{~mm}$ and the beams are considered of $\mathrm{ZnO}$ with $\hat{\rho}=5600 \mathrm{~kg} / \mathrm{m}^{3}, \mathrm{E}=40 \mathrm{GPa}, \mathrm{L}=1 \mu \mathrm{m}, \mathrm{N}=5 \times 10^{10} / \mathrm{m}^{2}$ and diameter $\mathrm{D}=0.1 \mu \mathrm{m}$.

Fig. 3 and Fig. 4 show the phase velocity of the first-order mode for magnetoelectrically open and shorted cases with different $N$, respectively. The calculation results for different number of microbeams are figured for a contrast. We can find the phase velocity decrease with the non-dimensional wavenumber, while increase with the number of the microbeams.

In order to show effect of the thickness of piezomagnetic layer, Fig. 5 and Fig. 6 show the phase velocity of the first-order mode for magnetoelectrically open and shorted cases, respectively. It can be found that the phase velocity increase with the thickness of piezomagnetic layer.

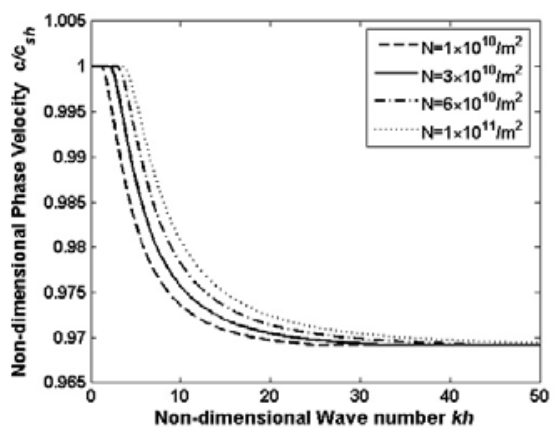

Figure.3 Dispersive relationship for magnetoelectrically open case with different $N$

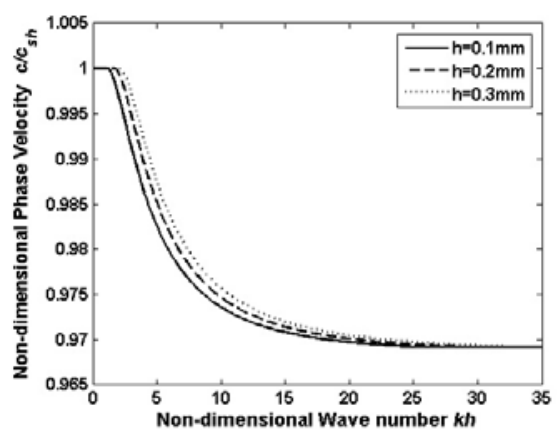

Figure.5 Dispersive relationship for magnetoelectrically open case with different $h$

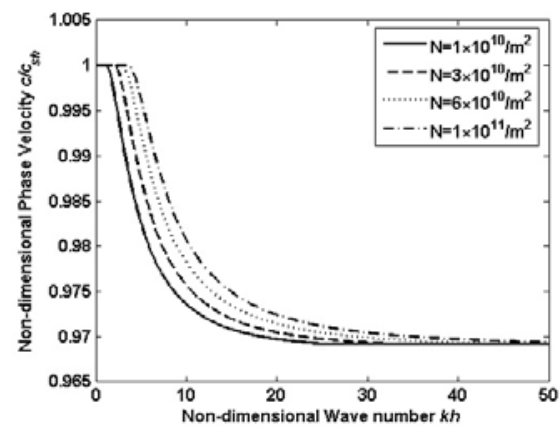

Figure.4 Dispersive relationship for magnetoelectrically shorted case with different $N$

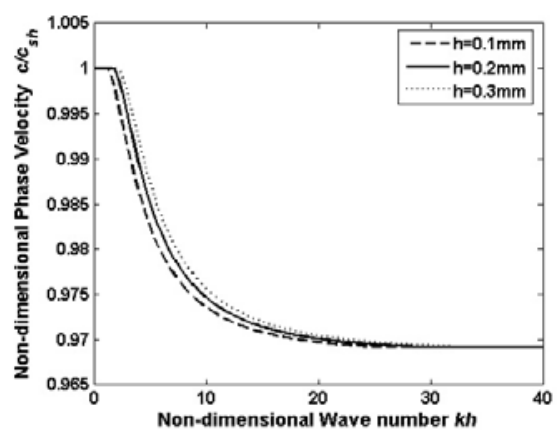

Figure.6 Dispersive relationship for magnetoelectrically shorted case with different $h$ 


\section{Conclusions}

SH surface acoustic waves propagation in layered piezomagnetic/piezoelectric structures covered a microbeam array are investigated in this paper. The solutions of dispersion relations are obtained with magnetoelectrically open or shorted conditions by analytical method. The effects of the microbeam on the phase velocity are presented and discussed in detail. From the results we can find that the effects of microbeams on the properties of SH-SAW are remarkable. The phase velocity decrease with the non-dimensional wave number, and increase with the thickness of piezomagnetic layer and the number of the microbeams. The analytical method and the results can be useful for the design of the resonators and sensors.

\section{Acknowledgements}

This research are supported by a grant from the Mechanics Initiative, a COE Program of Zhejiang Provincial Government in the Department of Mechanics and Engineering Science, Ningbo University (Grant No. xklx1508), Research Project of Ningbo University (No. XKL14D2071) and Ningbo Natural Science Foundation (No. 2014A610171) .

\section{References}

[1] C.W. Nan, Magnetoelectric effect in composites of piezoelectric and piezomagnetic phases, Physical Review B 50, 6082-6088 (1994)

[2] J. Ryu, A.V. Carazo, K. Uchino, H.E. Kim, Magnetoelectric properties in piezoelectric and magnetostrictive laminate composites, Japanese Journal of Applied Physics, Part 140 (2001) 4948-4951.

[3] L.L. Yuan, J.K. Du, T.F. Ma and J. Wang, Effect of viscous liquid on SH-SAW in layered magnetoelectric structures. Ultrasonics 53, 808-814, 2013.

[4] J.X. Liu, WeiYi Wei, DaiNing Fang,: Propagation behaviors on shear horizontal waves in piezoelectric-piezomagnetic periodically layered structure, Acta Mech. Solida Sin.23, 77-84(2010)

[5] X.D. Wang, C.J. Summers, Z.L. Wang, Large-scale hexagonal-patterned growth of aligned ZnO nanorods for nano-optoelectronics and nanosensor arrays, Nano Lett., Vol.4, (2004) 423-426.

[6] G. Ulises, L. Yirong, J. E. Gregory, A.S. Henry, Effect of ZnO nanowire morphology on the interfacial strength of nanowire coated carbon fibers, Compos. Sci. Technol., 71 (2011) 946-954.

[7] Z.Li, K. Feng, J. S. Yang, L. Tan, H. Lin, Collective buckling of nonuniform nanobeams interacting through an elastic substrate, Acta Mech 209, (2010) 285-293.

[8] H.J., Lin, Z.G. Chen, J. S. Yang, L. Tan, Effects of surface deformation on the collective buckling of an array of rigid beams on an elastic substrate, J. Mech Mater Struct, Vol. 5, No. 3, (2010) 495-506.

[9] N. Liu, , J.S. Yang, J. Wang, Shear Vibration of a Crystal Plate Carrying an Array of Microbeams, Philos. Mag. Lett., 91 (2011), 572-581.

[10] J.M. Gere, Mechanics of Materials, 5th ed., Books / Cole, Pacific Grove, CA, 2001.

[11] L. Meirovitch, Analytical Methodes in Vibrations, Macmillan, New York, 1967. 